\title{
El huerto ecopónico: un recurso efectivo para fomentar en los estudiantes una actitud proambiental y educación alimentaria
}

\author{
José Ramón Rivera Romero \\ jose.rivera@unicach.mx \\ https://orcid.org/0000-0003-3187-343X \\ Universidad de Ciencias y Artes de Chiapas, México
}

Martha Isela Baños Dorantes

martha isela80@outlook.com

https://orcid.org/0000-0001-5372-8642

Colegio de Bachilleres de Tabasco, Plantel 13,

Tabasco, México

Gladys Alatiel Moreno Santiago gladysmorenos45@dgetaycm.sems.gob.mx

https://orcid.org/0000-0002-7275-6902

Centro de Bachillerato Tecnológico

Agropecuario No. 45. Palenque, Chiapas, México

Dakar Lauriano Espinosa Jiménez

dakar.espinosa@unich.edu.mx

https://orcid.org/0000-0003-0476-6030

Universidad Intercultural de Chiapas, Sede Valle de Tulija, Salto de Agua, Chiapas, México

Ana Laura Luna Jiménez ana.luna.ujat@gmail.com https://orcid.org/0000-0003-3922-7002 Universidad Juárez Autónoma de Tabasco,

Campus Tenosique, Tabasco, México

Román Jiménez Vera roman.jimenez@ujat.mx

Universidad Juárez Autónoma de Tabasco,

Campus Tenosique, Tabasco, México https://orcid.org/0000-0002-5926-8592

Correspondencia: Nicolás González Cortés nicolas.gonzalez@ujat.mx

https://orcid.org/0000-0001-7336-4524

Universidad Juárez Autónoma de Tabasco,

Campus Tenosique, Tabasco, México 


\section{RESUMEN}

El objetivo fue implementar el huerto ecopónico como estrategia para mejorar la actitud proambiental y educación alimentaria en estudiantes de nivel primaria, secundaria, preparatoria y universidad en Tenosique Tabasco, México. El estudio tuvo una duración de siete meses, indicando la cosecha los primeros productos a los 70 días de haber implementado del huerto ecopónico. Para analizar el estado actual y cambios de nivel de actitud, se aplicó al inicio y al finalizar el estudio un cuestionario con 20 preguntas y cuatro niveles de respuesta. El cuestionario se dividió en tres categorías, 1) gusto de estar en contacto con la naturaleza $(\mathrm{CN}), 2)$ gusto por estudiar la naturaleza (EN) y 3 ) gusto por trabajar en la naturaleza (TN). Los resultados indicaron que al inicio del estudio, los estudiantes de mayor nivel educativo presentaban menor actitud proambiental y bajo interés por el cultivo del huerto. Sin embargo, al finalizar el estudio, los resultados indicaron un cambio positivo significativo en los cuatro niveles educativos, obteniendo los niveles más alto los estudiantes de primaria, seguido por estudiantes de secundaria, luego los de preparatoria y por último los universitarios. Se concluye que el huerto ecopónico en el patio de la escuela es un recurso efectivo para fomentar en los estudiantes una actitud proambiental y habilidades para la producción de alimentos.

Palabras claves: ecoeducación; actitud proambiental; seguridad alimentaria; tenosique. 


\title{
The ecoponic orchard: an effective resource to promote a pro-environmental attitude and food education in students
}

\begin{abstract}
The objective was to implement the ecoponic orchard as a strategy to improve a proenvironmental attitude and food education in primary, secondary, high school and university students in Tenosique Tabasco, Mexico. The study lasted seven months, with the harvest indicating the first products 70 days after implementing the ecoponic orchard. To analyze the current state and change in attitude level, a questionnaire with 20 questions and four response levels was applied at the beginning and at the end of the study. The questionnaire was divided into three categories, 1) liking to be in contact with nature $(\mathrm{CN}), 2)$ liking for studying nature $(\mathrm{EN})$ and 3 ) liking for working in nature $(\mathrm{TN})$. The results indicated that at the beginning of the study, the students with a higher educational level had less pro-environmental attitude and low interest in orcharding. However, at the end of the study, the results indicated a significant positive change in attitudes in the four educational levels, with the highest levels being obtained by primary school students, followed by secondary school students, then high school students and finally university students. It is concluded that the ecoponic garden in the schoolyard is an effective resource to promote in students a pro-environmental attitude and skills for food production.
\end{abstract}

Keywords: eco-education; pro environmental attitude; food safety; Tenosique.

Artículo recibido: 30 noviembre. 2021 Aceptado para publicación: 29 diciembre 2021 Correspondencia: nicolas.gonzalez@ujat.mx Conflictos de Interés: Ninguna que declarar 


\section{INTRODUCCIÓN}

En un mundo sobrepoblado, con más de 7 mil millones de habitantes, en combinación con la urbanización, cambios tecnológicos y otros factores, hacen que los estudiantes de todos los niveles educativos tengan menos contacto con la naturaleza y menor relación armónica entre el ambiente y sus actividades cotidianas (González, 2013). Estos factores impactan de forma negativa en: acciones en beneficio del medio ambiente, el bajo consumo de frutas/hortalizas y reducido interés por estudiar una carrera en programas educativos en las ciencias agropecuarias y naturales (biología, ecología, agronomía, veterinaria). Aunado a lo anterior se pronostica que para el año 2050, el $70 \%$ de la población vivirá en las ciudades, por tanto la producción de alimentos será todo un reto para el siglo XXI (Camberos, 2000 y Rodríguez et al., 2014).

Desde 1996, en la tercera Cumbre Mundial de Alimentos, organizada por la FAO (Organización de las Naciones Unidas para la Agricultura y la Alimentación) se habló de la seguridad alimentaria en los siguientes términos "Existe seguridad alimentaria cuando todas las personas tienen en todo momento acceso físico y económico suficientes alimentos inocuos y nutritivos para satisfacer sus necesidades alimentarias y sus preferencias en cuanto a los alimentos a fin de llevar una vida activa y sana" (FAO, 1996). Con este principio, se estableció por los países miembros de la FAO, reducir a la mitad el número de personas que padecen desnutrición crónica para el año 2015. Sin embargo, la misma FAO (2007a y 2007b) indicó que a pese a los avances en políticas de seguridad alimentaria en América Latina y el Caribe, el hambre y la desnutrición afectan a 52.5 millones de personas ( $9 \%$ de su población total); de estos son 9 millones de niñas y niños menores de cinco años que padecen desnutrición crónica. En esta parte del continente Americano, Guatemala es el país que registra la más alta prevalencia de niños con desnutrición, seguido de Honduras y Bolivia. Sin embargo, Brasil y México debido a su elevada densidad demográfica concentran más del $40 \%$ del total de casos de baja talla para la edad referida, con una prevalencia del $11 \%$ y $18 \%$, para Brasil y México respectivamente (CEPAL, 2006)

En México, la desnutrición crónica persiste como problema de salud pública entre la población (Rivera et al., 2013). Este problema se acentuará más, si se considera que en el año 2013 la población alcanzó 118.4 millones, durante este mismo año hubo 2.25 
millones de nacimientos, lo que implicó en términos absolutos un crecimiento de 1.58 millones de personas, y una tasa de crecimiento anual de 1.13 por ciento (CONAPO, 2013). Los estados con mayor inseguridad alimentaria identificada como moderada y severa son: Tabasco (49\%), Guerrero (44.0\%), Chiapas (38.9\%), Oaxaca (38.6\%) y Campeche (35.9\%). Se ha encontrado que entre mayor es la inseguridad alimentaria, mayor es la presencia de desnutrición infantil en los hogares (ENSANUT, 2013).

A pesar de los grandes esfuerzos para cubrir la demanda global de alimentos con los sistemas de producción convencional, no se ha reducido a cero la inseguridad alimentaria (FAO, 2012 y 2013). Sin embargo, la mayoría de los sistemas de producción agrícolas convencional impactan de forma negativa al medio ambiente y a los recursos naturales; por ejemplo, con el uso indiscriminado de agroquímicos contaminan los suelos, cuerpos de agua y el aire; afectando la salud del al hombre y la biodiversidad. Por otra parte, la SEMARNAT (2006) indica que con propósitos de expandir la superficie para actividades agropecuarias, más de la mitad de los bosques tropicales y templados del mundo ha desaparecido por lo que se talan entre 400,000 a 600,000 mil hectáreas por año; además, la mayoría de los suelos agrícolas están erosionados, y tres cuartas partes de las pesquerías del mundo se han agotado o se explotan a su máxima capacidad. Todos estos factores ponen en riesgo la seguridad alimentaria. Así mismo, la deforestación e incendios forestales son factores que acelera el calentamiento global; lo que traerá consigo sequias prolongadas en unos sitios y en otros lugres inundaciones más frecuentes: dos fenómenos bien conocidos que impactarán en la pérdida de cultivos y como resultado menos producción de alimentos.

Por tanto, para cubrir la seguridad alimentaria global para las actuales y futuras generaciones, los expertos en producción agropecuaria, deben diseñar e implementar agro tecnologías sustentables; es decir, sistemas de producción con el uso óptimo de los recursos naturales. Al respecto, el Instituto Internacional de Investigaciones sobre Políticas Alimentarias (IFPRI), reporta 11 innovaciones agro tecnológicas que pueden ayudar a la agricultura a enfrentar el reto de la seguridad alimentaria: protección de cultivos de malezas, insectos y enfermedades, sistemas de irrigación por goteo, tolerancia a la sequía, tolerancia al calor, manejo integrado de la fertilidad del suelo, siembra directa (sin labranza), eficiencia en el uso de nutrientes, agricultura orgánica, agricultura de precisión, riego por aspersión, y captación de agua (Agrosíntesis, 2015). Aunque estas 
agrotecnias están pensadas para cultivos como maíz, trigo y arroz, pueden ser empleadas para prácticamente cualquier otro cultivo, como es el caso de las hortalizas.

Con base a estos criterios de producción, Lara (1999) afirma que una estrategia sustentable para la producción de alimentos es la hidroponía. Por su parte Marulanda e, Izquierdo (2003), Zúñiga et al. (2004) y Castañeda (2001) describen que con esta tecnología se incrementan significativamente la cantidad y calidad de los alimentos hortícolas. Barbados (2009) y Morales et al. (2011) reportan que con la hidroponía se ahorra agua, fertilizantes y se contamina menos el ambiente, en comparación con los métodos de cultivo convencionales. Pero además, González (2012) describe que cuando se trabaja la biohidroponía con técnicas ecológicas como la biofertilización, control biológico de plagas/enfermedades, manejo integral de pequeñas especies pecuarias de traspatio y la aplicación del sistema 10R (reclutar, rechazar, reducir, reutilizar, restaurar, reciclar, rescatar, recompensar, responder y reforestar) se define como un sistema ecopónico, lo que significa literalmente huerto ecopónico (del griego aikos = casa y ponos = labor o trabajo) trabajar en armonía con nuestro planeta.

La ecoponia puede ser idónea para implementarse en los huertos familiares y escolares. Este sistema de producción de alimentos encaja muy bien con la filosofía de la FAO (2015), quien estableció en el marco del Foro Global Seguridad Alimentaria y Nutrición, que las escuelas y las universidades tienen un gran compromiso en fomentar el interés en los niños y jóvenes por la agricultura, además un mayor acceso a la educación y el conocimiento de nuevas formas empresariales basadas en la agricultura, ayuda a los jóvenes a convertirse en una fuerza vital para la innovación en la agricultura familiar, aumentando la disponibilidad de alimentos, los ingresos y el bienestar de agricultores y de la comunidad. Además la FAO recomendó que los programas de estudio de todos los niveles educativos debe abordar cuestiones teóricas y prácticas de agroecología, con el fin de transmitir vocación hacia la agricultura como opción socioeconómica; de lo contrario los jóvenes, inclusive los de comunidades agrícolas, se irán alejando de las actividades del campo en busca de un empleo en las grandes ciudades.

Además la escuela, como tal, es parte fundamental de una sociedad de la información que lleva a la sociedad a mejores niveles de equidad y a una mejor trasformación con el medio ambiente natural, esto a través de modelos pedagógicos y andragógicos basados en valores sociales, humanos y ecológicos (Bueno, 2010). Por tanto, los huertos ecopónicos 
pueden constituirse en escenarios para el aprendizaje significativo, al proporcionar recursos didácticos que al implementarse en las escuelas bajo objetivos precisos pueden fomentar en los estudiantes una sólida educación alimentaria y ambiental. Por tanto, el objetivo fue implementar huertos ecopónicos en las escuelas como una estrategia para conocer la percepción pro ambiental y alimentaria en estudiantes de diferentes niveles educativos.

\section{MATERIALES Y MÉTODOS}

El presente estudio se desarrolló en cuatro escuelas piloto del municipio de Tenosique Tabasco, México; municipio que colinda con Guatemala, localizado en la región de los Ríos, al sur del Estado (Figura 1). El clima es cálido húmedo con abundantes lluvias en verano, con temperatura media anual de $30.5^{\circ} \mathrm{C}$ y la mínima absoluta se mueve entre los $28.4^{\circ} \mathrm{C}$. La precipitación es de 3,286 mm con un promedio máximo mensual de $400 \mathrm{~mm}$ en el mes de septiembre y un mínimo mensual de $50 \mathrm{~mm}$ en el mes de abril. Las mayores velocidades del viento se registran en los meses de noviembre, con velocidades que alcanzan los $30 \mathrm{~km} \cdot \mathrm{h}^{-1}$ (INEGI, 2005).

Figura 1. Localización y ubicación del municipio de Tenosique, Tabasco, México, donde se ubican las escuelas piloto para el establecimiento de huertos ecopónicos.

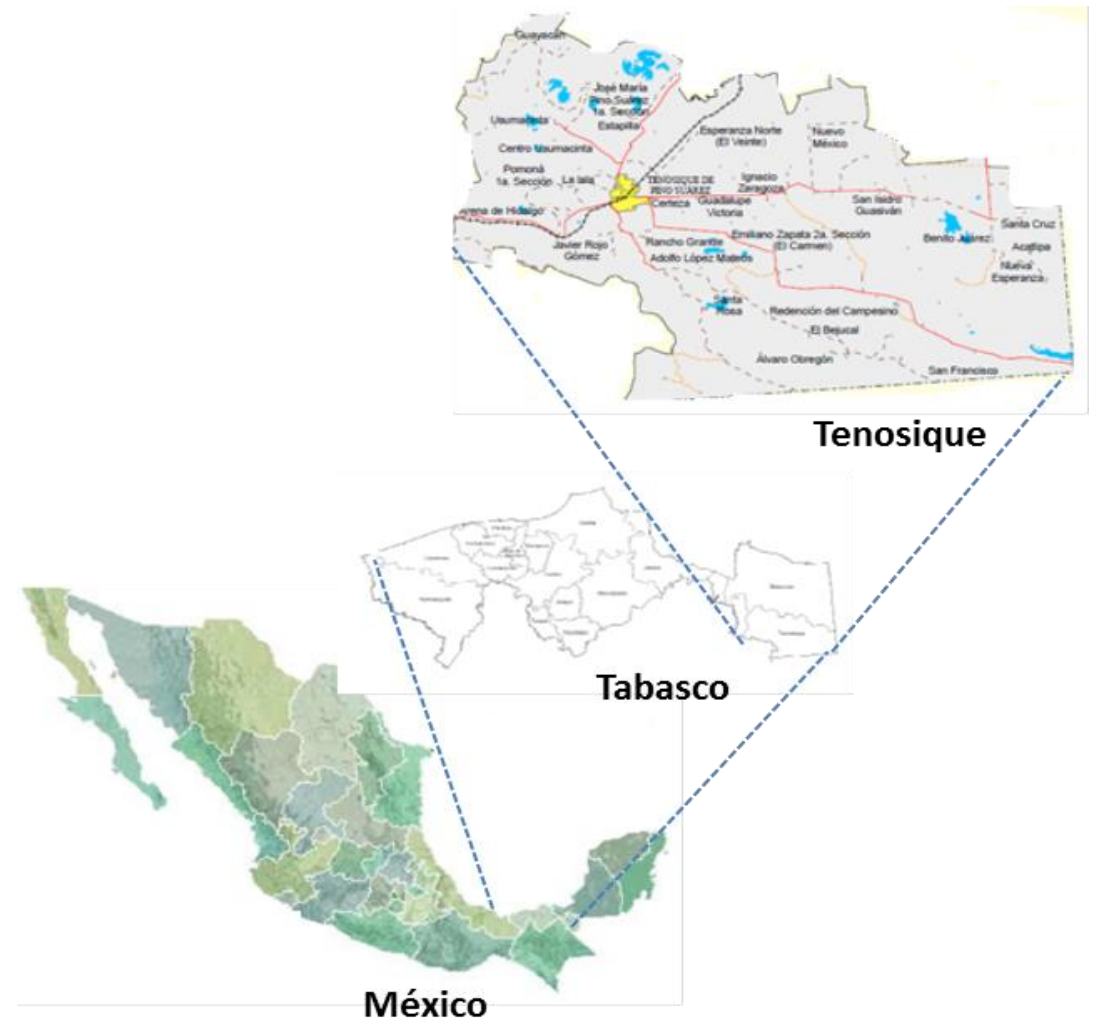


Se seleccionaron cuatros escuelas: dos de nivel básico (primaria y secundaria) una de medio superior (bachillerato) y una de nivel superior (universidad). La selección se basó en la respuesta, disponibilidad e interés mostrado por los directores de las escuelas hacia el desarrollo del proyecto. Después se seleccionaron los grupos de estudiantes, se les dio preferencia a los cursaban una asignatura relacionada a las ciencias naturales, biología, ecología y cultura ambiental. Después se instruyó al profesor y su grupo de alumnos sobre temas selectos del cultivo de huertos ecopónicos y crianza de pequeñas especies pecuarias.

Figura 2. Capacitación de maestros y alumnos en el cultivo de hortalizas y crianza de pequeñas especies pecuarias.

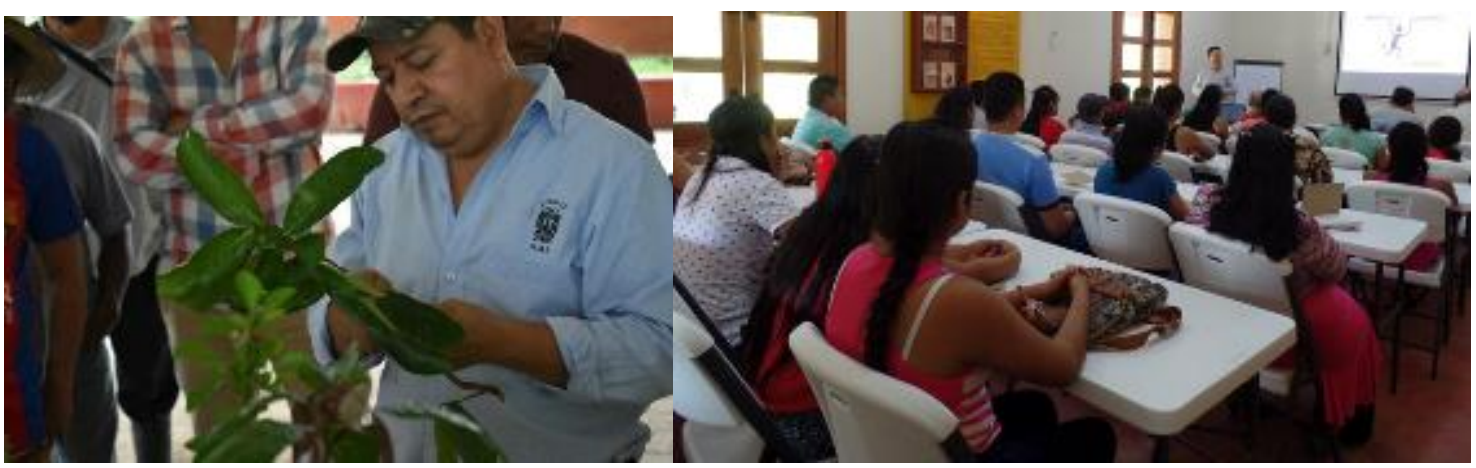

A continuación se mencionan los nombres de los centros escolares que participaron, el nombre de la asignatura en la que se insertó el proyecto, año o semestre que cursaban y el número de alumnos agrupados por género.

Tabla 1. Escuelas que participaron en el proyecto huertos ecopónicos.

\begin{tabular}{|c|c|c|c|c|c|c|}
\hline Nivel & Nombre & Materia & $\begin{array}{c}\text { Año / } \\
\text { semestre }\end{array}$ & $\begin{array}{r}\text { Alumno } \\
\text { Masculino }\end{array}$ & $\begin{array}{l}\text { s/Género } \\
\text { Femenino }\end{array}$ & Total \\
\hline Primaria & Carlos Pellicer Cámara & $\begin{array}{l}\text { Ciencias } \\
\text { Naturales }\end{array}$ & 3er. Año & $16(51 \%)$ & $15(49 \%)$ & 31 \\
\hline Secundaria & Gustavo Díaz Ordaz & Biología & $\begin{array}{l}\text { 2do. } \\
\text { Año }\end{array}$ & $12(43 \%)$ & $14(57 \%)$ & 26 \\
\hline Bachillerato & $\begin{array}{l}\text { Centro de Bachillerato } \\
\text { Tecnológico, Industrial } \\
\text { y de Servicios (CBTIS) } \\
249\end{array}$ & Ecología & 2do. año & $21(49 \%)$ & $22(51 \%)$ & 43 \\
\hline Universidad & $\begin{array}{l}\text { División Académica } \\
\text { Multidisciplinaria de los } \\
\text { Ríos (UJAT) }\end{array}$ & $\begin{array}{l}\text { Cultura } \\
\text { ambiental }\end{array}$ & $\begin{array}{c}2 \text { do. } \\
\text { Semestre }\end{array}$ & $19(46 \%)$ & $22(54 \%)$ & 41 \\
\hline
\end{tabular}


Después se diseñó e implantó un el huerto ecopónico a nivel suelo, aéreos o tipo hidropónico de cuatro niveles, elaborado con material de acero, malla hexagonal y cuadriculada, tubos de PVC de 6 pulgadas o botes de 19 L reciclados de las plantas purificadoras de agua, estos últimos se partieron a la mitad de forma longitudinal con el uso de serrucho. Las dimensiones del prototipo fueron de $2 \mathrm{~m}$ de largo, $1.60 \mathrm{~m}$ de alto y $0.60 \mathrm{~m}$ de ancho (Figura 3). En la parte baja del prototipo se colocaron gallinas, en el segundo nivel se instalaron pollos, en el tercer nivel conejos y en el último nivel codornices. A demás en cada nivel hubo tubos de PVC se cultivó tomate (Solanum lycopersicum), diversos especies de chiles (Capsicum annum), y plantas aromáticas/medicinales: menta (Mentha piperita), tomillo (Thymus vulgaris), orégano (Origanum vulgare) y epazote (Chenopodium Ambrosioides). El control de plagas se realizó con extractos etílicos de neem (Azadirachta indica) y orégano orejón (Plectranthus amboinicus) previamente elaborados en los talleres de frutas de la DAMR, aunado durante el proyecto se aplicó el sistema 10R descrito por González (2012).

Figura 3. Prototipos de huerto ecopónico en escuelas de Tenosique, Tabasco México

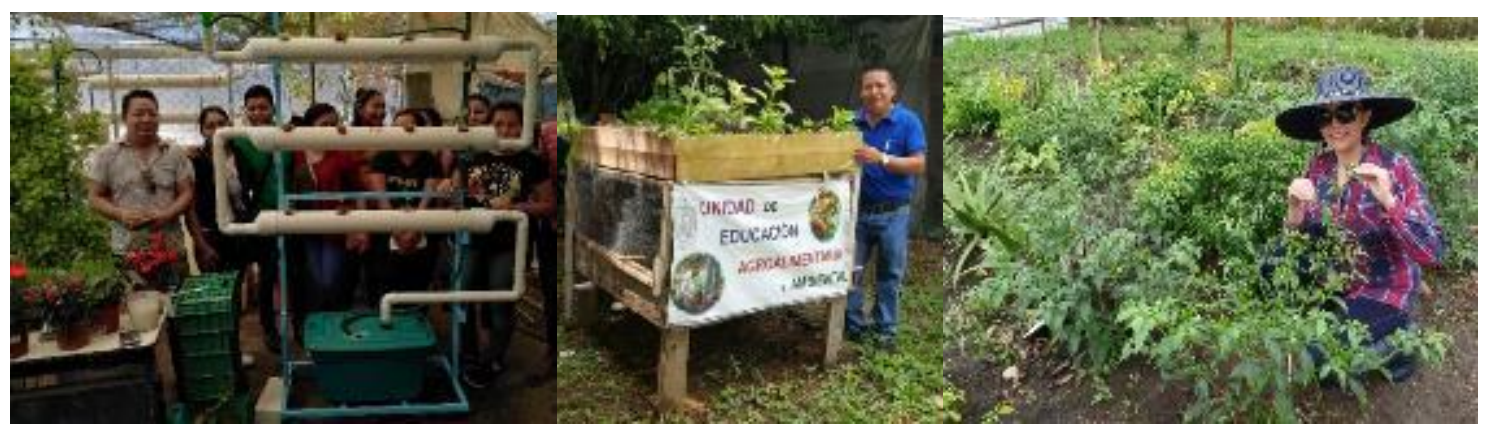

(Diseño propio).

Para el cultivo de las hortalizas se utilizó una mezcla de tierra de campo y composta (elaborada con bagazo de caña de azúcar y hojas y peciolos troceados en partículas pequeñas de palma de aceite) en una relación de 1:1 (v/v).

Figura 4. Preparación del sustrato para la siembra de semillas de hortalizas.

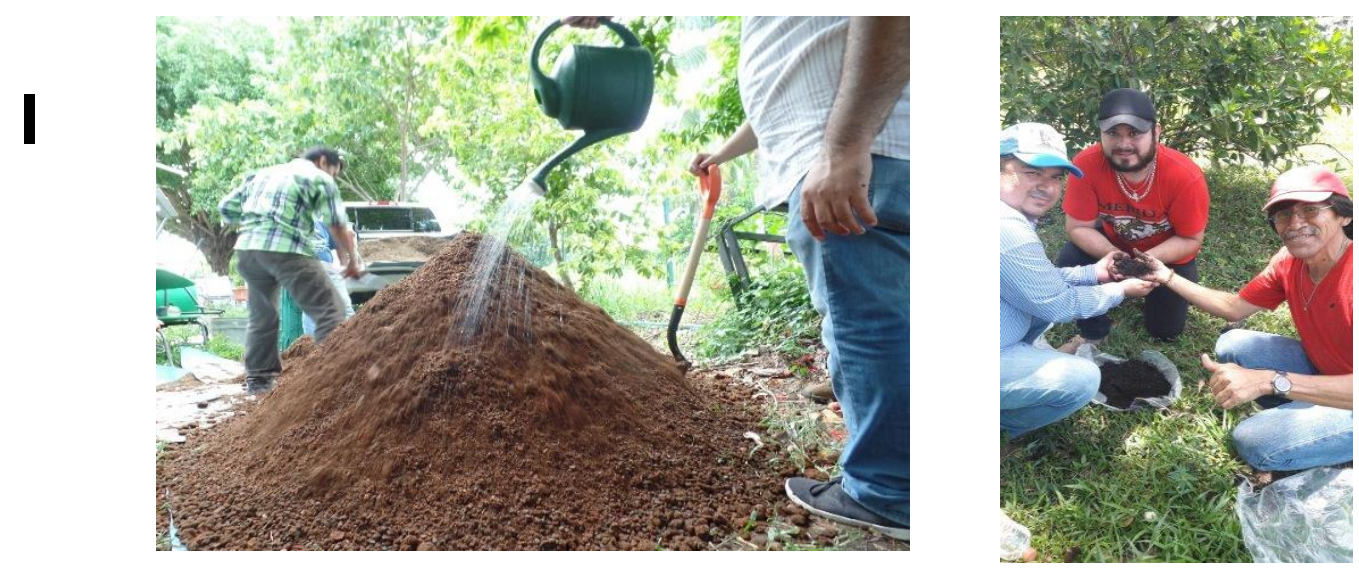


Los alumnos se encargaron del riego de las hortalizas y del control de insectos plaga de forma manual y con extractos de neem y orégano, bajo la tutela de los técnicos del proyecto. De igual manera los alumnos alimentaban los semovientes a libre acceso con una mezcla, previamente elaborada en el taller de alimentos de la DAMR, de maíz amarillo (Zea mays), sorgo (Sorghum spp.) y caña de azúcar (Saccharum spp.) todos molidos a un tamaño de partícula menor de $2 \mathrm{~mm}$ y mezclado con alimento comercial, en una relación de 30:70 (p/p).

Figura 5. Germinación de semillas en diferentes materiales reutilizados.

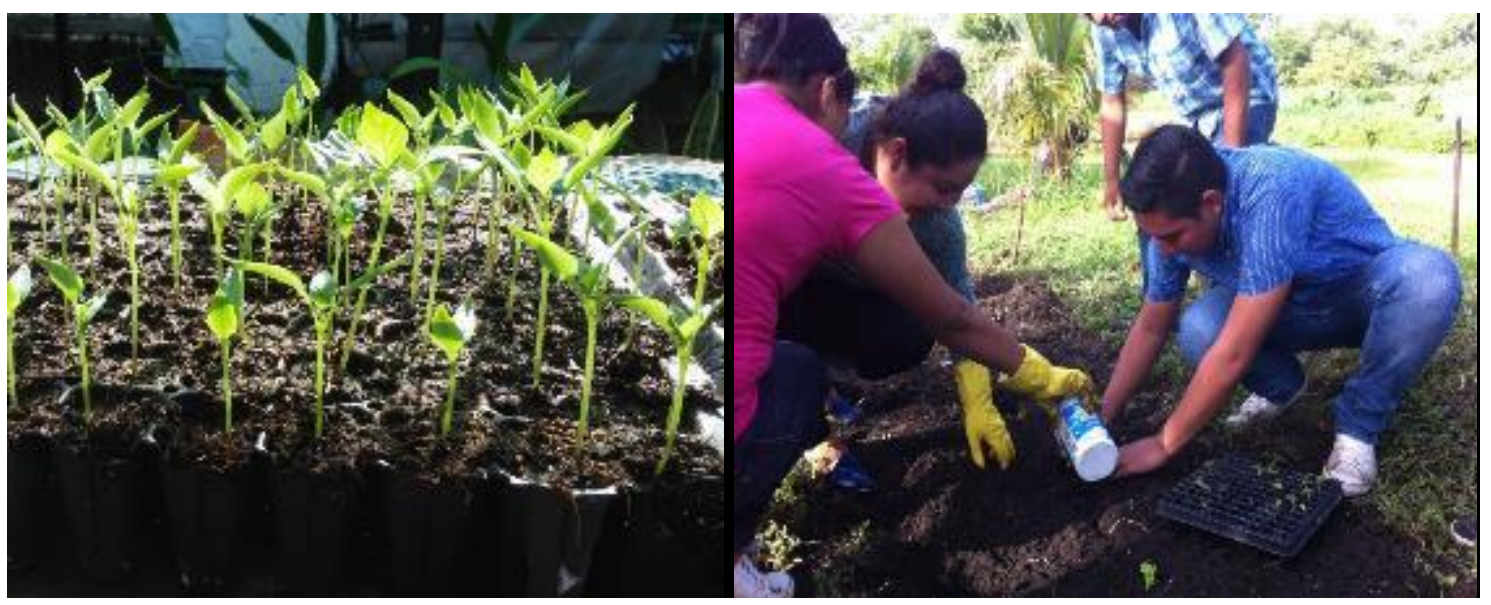

Los resultados obtenidos en la producción se evaluaron a partir de los 70 días de la siembra de hortalizas. Las codornices empezaron a producir huevos a los 60 días y las gallinas a los seis meses. La producción obtenida fue repartida entre los estudiantes. En el caso de la escuela primaria, la producción se destinó para el comedor escolar.

Figura 6. Manejo del huerto ecopónico implementado en las escuelas

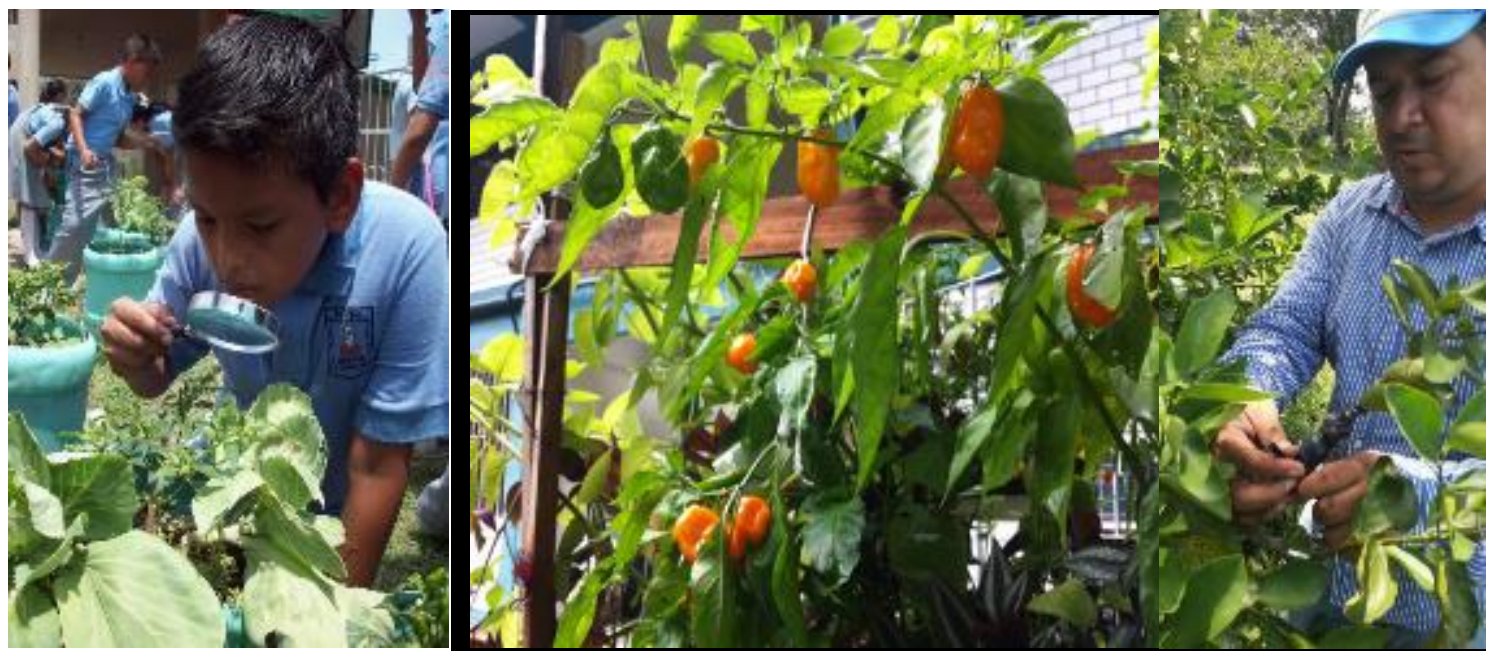


Para identificar el estado actual y cambio en el nivel de actitud (pro ambiental y alimentario) en torno al empleo de esta estrategia ecopónica, a todos los estudiantes participantes se les aplicó (al inicio y al finalizar el proyecto) un cuestionario adaptado de Kibert (2000) y Sosa et al. (2010). El cuestionario constó de 20 preguntas y tenía 4 niveles de respuesta: No me gusta (0), Me gusta poco (1), Me gusta (2) y Me gusta mucho (3). El cuestionario se dividió en tres categorías, la primera categoría fue integrada por 4 preguntas enfocadas por el gusto de estar en contacto con la naturaleza $(\mathrm{CN})$, fue integrada por 4 preguntas, al multiplicar el número de preguntas y el valor de la respuesta se formó un índice de 0 a 12, esto al considerar 4 (número de preguntas) por 3 (me gusta mucho) igual a 12. La segunda categoría constó de 10 preguntas dirigidas por el gusto por estudiar la naturaleza (EN), el índice vario de 0 a 30, y en la tercera categoría se formó con 6 preguntas orientadas por el gusto de trabajar con el cultivo de hortalizas y producción pecuaria (TN), y el índice fue de 0 a 18 . Los datos de cada una de las categorías fueron convertidos aritméticamente a una escala del 0 al 10 al que se llamó ecoíndice. Además, el cuestionario contenía una breve portada en la que los estudiantes informaban su género y se consideró como variable independiente. El cuestionario se aplicó antes y al final el estudio.

\section{RESULTADOS Y DISCUSIÓN}

Como se puede observar en el la Tabla 2, el huerto ecopónico tuvo un efecto importante en fomentar educación pro ambiental y alimentaria en los cuatro niveles educativos. Al inicio del estudio se observó que los alumnos de nivel Primaria tenían una actitud ambiental y alimentaria por arriba de 7, en una escala del 0 al 10; esto coincide con Shore (1996) quien describe que como producto de la evolución, la especie humana se ha dotado de un "cerebro ecológico". En contraste, los varones de nivel preparatoria y universidad, al inicio indicaron tener una actitud ambiental y alimentario menor de 6; esto quiere decir no les interesaba estar en contacto con la naturaleza, estudiar la naturaleza ni realizar actividades agropecuarias. Este desinterés se observó claramente en respuesta a una de las preguntas que indicaba que dibujaran el lugar en donde realizan las actividades que más les gusta, la mayoría de los niños se dibujaron jugando en el centro de un escenario natural, donde resaltaban dibujos de árboles frondosos, un sol pintado de amarillo, un cielo azul, aves, reptiles, etc. En contraste, la mayoría de los jóvenes de preparatoria y universitarios se dibujaron en un escenario solitario; acostados en la cama viendo TV, en 
la hamaca con sus audífonos y celular, o usando la computadora. Esto coincide con Pearce y Turner (1997) quien menciona que las percepciones primarias con la vinculación a la tierra que se desarrollan en la infancia tienden a desaparecer si no hay entrada de conocimientos socio-emocional sensible y congruente intelectualmente. Bajo este enfoque, Wilson (1992) aplica dos temimos; biophilia que significa amor por la naturaleza, pero describe que si este atractivo natural no se estimula y se pierde se dará el fenómeno de biophobia; que la define como una aberración por la naturaleza, y que los humanos la manifiestan cuando consideran la naturaleza como "explotar los recursos naturales para la producción de bienes y servicios para el confort", sin tomar en cuenta el deterioro ambiental. Al final del estudio, se notó un cambio significativo de actitud en los varones de preparatoria y universidad. Sin embargo, el nivel delos niños de primaria el nivel de actitud fue superior con 8.50, y para las niñas de 8.25.

Tabla 2. Cambio de actitud pro ambiental y alimentaria en estudiantes de diferentes niveles educativos con el manejo del huerto ecopónico.

\begin{tabular}{lccc}
\hline \multicolumn{1}{c}{ Alumnos } & Género & Ecoíndice Inicial & Ecoíndice Final \\
\hline Primaria & Niños & $7.40 \pm 0.98$ & $8.50 \pm 0.48$ \\
Necundaria & Niñas & $7.20 \pm 0.96$ & $8.25 \pm 0.39$ \\
& Niños & $6.80 \pm 1.20$ & $7.90 \pm 0.47$ \\
Bachiller & Niñas & $6.20 \pm 1.12$ & $7.35 \pm 0.41$ \\
& Hombres & $5.95 \pm 1.86$ & $7.58 \pm 0.52$ \\
Universidad & Mujeres & $6.10 \pm 1.52$ & $7.20 \pm 0.53$ \\
& Hombres & $5.80 \pm 1.48$ & $7.46 \pm 0.41$ \\
& Mujeres & $6.10 \pm 1.22$ & $7.17 \pm 0.43$ \\
\hline
\end{tabular}

\pm Desviación estándar.

El género femenino tiene mayor interés por estar en contacto con la naturaleza, poco gusto por estudiar temas relacionados con la naturaleza y menos realizar actividades agropecuarias. En los niños y hombres quienes indicaron tener mayor gusto por estar in situ, estudiando y trabajando en campo abierto; indicaron que les interesa cultivar hortalizas y la producción pecuaria. Esto coincide con los datos de la ANUIES reportados por el IPN (2006) donde indican que en las carreras de agronomía y veterinaria en su mayoría son hombres que cursan estas carreras, en una relación de 3 a 1; aunado son carreras con baja matrícula, por lo que recomiendan establecer estrategias para fomentar 
estos programas con los niños; ya que son muy importantes estos programas de estudio para la producción de alimentos. En otro estudio reportado por Fraj y Martínez (2005) encontraron que la actitud medioambiental está relacionada con el conocimiento y habilidades medioambientales. Otro trabajo por Cerda et al., (2007) analizaron el perfil y la conducta ambiental de alumnos universitarios, y determinaron que los alumnos tienen un comportamiento negativo frente al medio ambiente.

\section{CONCLUSIONES}

El ecoíndice de educación ambiental y alimentaria en los estudiantes de los cuatro niveles educativos mejoró con respecto al inicio. El huerto ecopónico es una estrategia interactiva, donde los profesores pueden enseñar de una manera dinámica y divertida diferentes áreas del conocimiento. Por su parte, los estudiantes de los diferentes niveles educativos tienen la oportunidad de jugar/aprender con la naturaleza y promover acciones amigables entre el medio natural y las actividades de los programas educativos. Con el huerto ecopónico los alumnos conocen diferentes formas para la producir alimentos de un amanera amigable al ambiente. Por tanto, se invita a los actores escolares a implementar el huerto ecopónico en escuelas rurales y urbanas, para el cultivo de una amplia variedad de hortalizas, plantas aromáticas, medicinales y ornamentales, así como la producción de pequeñas especies pecuaria de traspatio. Desde el aula y el patio de la escuela se puede fomentar en los estudiantes en una sólida educación ambiental y alimentaria para un mejor futuro de las actuales y futuras generaciones.

\section{LISTA DE REFERENCIAS}

Agrosíntesis, (2015). Agro tecnologías pueden incrementar la productividad en el campo, reducir el precio de los alimentos y el hambre. En línea: http://www.agrosintesis.com/component/content/article/49-front-page/905-agrotecnologias-pueden-incrementar-la-productividad-en-el-campo-reducir-elprecio-de-los-alimentos-y-el-hambre

Barbados, J. L. (2009). Hidroponía: su empresa de cultivo en agua. Edit. Albatros. Argentina. $190 \mathrm{p}$.

Bueno, M. 2010. Manual práctico del huerto ecológico. La fertilidad de la tierra Ediciones. España. 306 p.

Camberos, C. M. (2000). La seguridad alimentaria de México en el año 2030. Revista Ciencia Ergo Sum. Universidad Autónoma del Estado de México. 7(1): 49-55 p. 
Castañeda F. 2001. Manual técnico de hidroponía popular. INCAP, Guatemala. En línea: http://www.bvssan.incap.org.gt/

Cerda, U. A., García P. L., Díaz M. M. y Núñez N.C. (2007). Perfil y conducta ambiental de los estudiantes de la universidad de Talca, Chile. Panorama Socioeconómico, 25(035):148-159.

CEPAL, (Comisión Económica para América Latina y el Caribe) (2006). Desnutrición infantil en América Latina y el Caribe. DESAFIOS: Boletín de la infancia y adolescencia sobre el avance de los objetivos del desarrollo del milenio. Vol. 2.

CONAPO, (Comisión Nacional de Población). (2013). La Situación Demográfica de México 2013. Disponible en línea: http://www.conapo.gob.mx/

ENSANUT, (Encuesta Nacional de Salud y Nutrición). (2013). Resultados por entidad federativa. Tabasco. Primera edición electrónica. Disponible en línea: http://ensanut.insp.mx/informes/Tabasco-OCT.pdf

FAO, (Organización de las Naciones Unidas para la Agricultura y la Alimentación). (1996). Cumbre Mundial sobre la alimentación. Disponible en línea: http://www.fao.org/docrep/003/w3548s/w3548s00.htm

FAO, (Organización de las Naciones Unidas para la Agricultura y la Alimentación). $\left(2007^{\mathrm{a}}\right)$. Cambio climático y seguridad alimentaria: un documento marco. Roma, Italia. En línea: http://bvssan.incap.int/local/cambio-climatico/CAMBIOCLIMATICO-INSAN-MARCO-FAO.pdf

FAO, (Organización de las Naciones Unidas para la Agricultura y la Alimentación). (2007b). Crear y manejar el huerto escolar. Roma, Italia. 210 p.

FAO, (Food and Agriculture Organization United Nations). (2012). The state food insecurity in the world: Economic growth is necessary but not sufficient to accelerate reduction of hunger and malnutrition. En línea http://www.fao.org/docrep/016/i3027e/i3027e.pdf

FAO, (Food and Agriculture Organization of the United Nations). (2013). Food security and nutrition in Mexico An overview. En línea: http://coin.fao.org/coinstatic/cms/media/2/13843839442340/overview_food_security_and_nutrition_in_ mexico_2013-prov.pdf.

FAO, (Organización de las Naciones Unidas para la Agricultura y la Alimentación). (2015). Plan para la seguridad alimentaria, nutrición y erradicación del hambre de 
la

En

línea:

http://www.fao.org/fileadmin/user_upload/rlc/docs/celac/ESP_Plan_CELAC_2 025.pdf. Fraj, A. E. y E. Martínez S. (2005). El nivel de conocimiento medioambiental como factor moderador de la relación entre la actitud y el comportamiento ecológico. Investigaciones Europeas de Dirección y Economía de la Empresa Universidad de Zaragoza. 11(1):223-243.

González C. N. (2012). El huerto ecológico y el sistema 10R: un recurso didáctico para fomentar educación ambiental en la escuela. Revista Diálogos 39(1): 14-18.

González C.N. (2013). El huerto escolar = Educación + Alimentación + Medio ambiente: Una alternativa para favorecer la seguridad alimentaria y afrontar el cambio climático. Universidad Juárez Autónoma de Tabasco. Villahermosa, Tabasco México. 120 p.

INEGI, (Instituto Nacional de Estadística, Geografía e Informática). (2005). Prontuario de información geográfica municipal de los Estados Unidos Mexicanos, Tenosique, Tabasco. Clave geoestadística 2017. En línea: http://www3.inegi.org.mx/sistemas/mexicocifras/datosgeograficos/27/27017.pdf

IPN, (Instituto Politécnico Nacional). (2006). ¿Cómo estamos en educación superior? Innovación Educativa. 6(31)1-9.

Kibert, N.C. (2000). An analysis of the correlations between attitude, behavior and knowledge components of environmental literacy in undergraduate university students. Master of Science Thesis. University of Florida.

Lar,a H. A. (1999). Manejo de la solución nutritiva en la producción de tomate en hidroponía. Terra Latinoamericana 7(3) 221-229.

Morales, P. J. A., I. Miranda V., I Gil V., y A. Bastida T. (2011). Introducción a la hidroponía. Universidad Autónoma Chapingo. 169 p.

Marulanda, C. E. y J. Izquierdo. (2003). Manual Técnico: La huerta hidropónica popular. Organización de las Naciones Unidas para la Agricultura y la Alimentación (FAO) y Programa de las Naciones Unidas para el Desarrollo (PNUD), $3^{\text {a }}$. Edic. Santiago, Chile132 p.

Pearce, D. y Turner, W. (1995). Economía de los recursos naturales y del medio ambiente. Celeste Ediciones-Colegio de Economistas de Madrid. Madrid, España. 
Rivera, D. J. A.; Cuevas N. L.; González de C. T.; Shamah L. T. y García F. R. (2013). Desnutrición crónica en México en el último cuarto de siglo: análisis de cuatro encuestas nacionales. México. Salud Pública de México, 55(2):161-169.

Rodríguez, M. S. J, M. García G. y P. Pérez C. (2014). Producir alimentos: reto ante a una crisis. In: González C. N., et al. (Comp.). Investigaciones en Ciencias Biológicas. Tomo I. Universidad Juárez Autónoma de Tabasco, Villahermosa, Tabasco, México. pp. 44-63.

SEMARNAT, (Secretaria del Medio Ambienta y Recursos Naturales). (2006). Estrategia de educación ambiental para la sustentabilidad en México., México, D. F. 255 pp.

Sánchez-del Castillo, F., E. del C. Moreno-Pérez, e. 1. Cruz-Arellanes. (2009). Producción de jitomate hidropónico bajo invernadero en un sistema de dosel en forma de escalera. Revista Chapingo. Serie Horticultura, 15(1) 67-73.

Sosa, S. B., I. Márquez R., A. Eastmond, E. Ayala M. y A. Arteaga M. (2010). Educación superior y cultura ambiental en el sureste de México. Universidad y Ciencia. 26(1) $1-18$.

Shore, B. 1996. Culture in mind: cognotion, culture, and the problem of meaning. Oxford University Press, Inc. ISBN 0-19-509597-9.

Wilson, E. O. (1992). The diversity of life. Cambridge, MA: Harvard University Press. Zúñiga-Estrada, L.; J. de J. Martínez-Hernández; G. A. Baca-Castillo; Á. MartínezGarza, J. L. Tirado-Torres y J. Kohashi-Shibata (2004). Producción de chile pimiento en dos sistemas de riego bajo condiciones hidropónicas. Agrociencia, 38(2) 207-218. 